\title{
Diphoton Amplitudes in Three-Loop Quantum Chromodynamics
}

\author{
Fabrizio Caola, ${ }^{1,2, *}$ Andreas von Manteuffel@ ${ }^{3, \dagger}$ and Lorenzo Tancredi $\odot^{1, *}$ \\ ${ }^{1}$ Rudolf Peierls Centre for Theoretical Physics, University of Oxford, Clarendon Laboratory, \\ Parks Road, Oxford OX1 3PU, United Kingdom \\ ${ }^{2}$ Wadham College, University of Oxford, Parks Road, Oxford OX1 3PN, United Kingdom \\ ${ }^{3}$ Department of Physics and Astronomy, Michigan State University, East Lansing, Michigan 48824, USA
}

(Received 9 December 2020; accepted 9 February 2021; published 18 March 2021)

\begin{abstract}
We consider the three-loop scattering amplitudes for the production of a pair of photons in quarkantiquark annihilation in QCD. We use suitably defined projectors to efficiently calculate all helicity amplitudes. We obtain relatively compact analytic results that we write in terms of harmonic polylogarithms or, alternatively, multiple polylogarithms of up to depth three. This is the first calculation of a three-loop four-point scattering amplitude in full QCD.
\end{abstract}

DOI: 10.1103/PhysRevLett.126.112004

Multiloop scattering amplitudes in quantum chromodynamics (QCD) are a crucial ingredient for the precision physics program carried out at particle colliders such as the Large Hadron Collider at CERN. When combined with the corresponding real radiation, multiloop scattering amplitudes make it possible to compute high-precision predictions for a multitude of key processes. In the recent past, such calculations played a fundamental role for the Higgs characterization [1], for the extraction of fundamental parameters of the standard model (SM) [2,3], and for the study of electroweak bosons [4-6] and the top quark [7], to mention a few examples. These investigations allow for a deep scrutiny of the SM, which is essential for establishing its validity and for revealing possible tensions pointing toward new physics.

Together with their importance for particle collider phenomenology, scattering amplitudes in QCD also provide us with an important laboratory to investigate formal properties of the perturbative expansion of realistic quantum field theories (QFTs). With the increase of the perturbative order, one faces the computation of increasingly complicated multiloop Feynman diagrams that can depend on multiple scales according to the number of particles involved in the collision. This translates into increasingly involved analytic properties of the special functions required to express the corresponding scattering amplitudes in closed analytic form. The calculation of multiloop scattering amplitudes have helped reveal intricate and fascinating mathematical structures, whose further

Published by the American Physical Society under the terms of the Creative Commons Attribution 4.0 International license. Further distribution of this work must maintain attribution to the author(s) and the published article's title, journal citation, and DOI. Funded by SCOAP ${ }^{3}$. investigation has the potential to improve our understanding of perturbative QFT.

For these reasons, in the last two decades a considerable effort has been devoted to push SM calculations further. As a result, an impressive set of techniques have been developed that have made it possible to compute most $2 \rightarrow 2$ scattering amplitudes up to two loops in massless QCD for many processes relevant at hadron colliders [8-21] and also to include massive effects approximately and exactly [22-30]. Recently, also the first results for twoloop amplitudes for $2 \rightarrow 3$ scattering processes in QCD have been obtained [31-41], opening the way to the first $2 \rightarrow 3$ next-to-next-to-leading-order (NNLO) studies at the Large Hadron Collider [42,43].

In parallel to the effort to overcome the barrier of twoloop scattering amplitudes for $2 \rightarrow 3$ processes, similar work is required to push the calculation of $2 \rightarrow 2$ QCD processes up to three loops. Indeed, while the first $2 \rightarrow 2$ three-loop results have appeared in $\mathcal{N}=4$ Super YangMills [44] and in $\mathcal{N}=8$ Super Gravity [45], three-loop calculations in realistic QFTs have only been performed for simple $2 \rightarrow 1$ processes [46-48]. Only recently have some of the ingredients for obtaining three-loop $2 \rightarrow 2$ amplitudes in massless QCD started to appear [49,50].

In this Letter, we document the calculation of three-loop QCD corrections to the scattering amplitude for diphoton production in quark-antiquark annihilations. This is the first three-loop amplitude for a $2 \rightarrow 2$ scattering process in fullcolor QCD. The $q \bar{q} \rightarrow \gamma \gamma$ process is arguably a very natural place to start the investigation of three-loop corrections to $2 \rightarrow 2$ scattering in a realistic QFT. Indeed, these amplitudes involve only massless external and virtual particles and, moreover, have a much simpler color structure than the corresponding amplitudes for the production of colored partons. The relevant one-loop and two-loop amplitudes have been known for a long time $[10,12]$ and followed by a 
multitude of phenomenological studies up to NNLO in QCD [51-53]. However, we would like to stress that despite the aforementioned simplifications, this process still contains all of the analytic complexity of a generic massless $2 \rightarrow 2$ scattering process. Hence, we expect that the results obtained here can be extended to compute all three-loop scattering amplitudes for the production of two massless partons in hadronic collisions.

We now describe our calculation. We consider the process

$$
q\left(p_{1}\right)+\bar{q}\left(p_{2}\right)+\gamma\left(p_{3}\right)+\gamma\left(p_{4}\right) \rightarrow 0
$$

and obtain its physical equivalent $q \bar{q} \rightarrow \gamma \gamma$ via crossing symmetry $p_{3,4} \rightarrow-p_{3,4}$. We parameterize the kinematics of Eq. (1) by defining the usual Mandelstam invariants:

$$
\begin{gathered}
s=\left(p_{1}+p_{2}\right)^{2}, \quad t=\left(p_{1}+p_{3}\right)^{2}, \quad u=\left(p_{2}+p_{3}\right)^{2}, \\
p_{1}^{2}=p_{2}^{2}=p_{3}^{2}=p_{4}^{2}=0, \quad \text { and } \quad s+t+u=0 .
\end{gathered}
$$

We also introduce the dimensionless variable $x=-t / s$ such that, in the physical scattering region, one has

$$
s>0, \quad t<0, \quad \text { and } \quad u<0 \Rightarrow 0<x<1 .
$$

We use the helicity of the incoming quark $\lambda_{q}$ and of the incoming photons $\lambda_{3,4}$ to label the scattering amplitude of the process. We denote the scattering amplitude between well-defined helicity states by $\mathcal{A}_{\lambda_{q} \lambda_{3} \lambda_{4}}$. Using parity, chargeconjugation, and symmetry relations, it is easy to see that there are only two independent helicity configurations $[10,12]$. In what follows, for clarity we will compute the overcomplete set of four helicity configurations $\left\{\lambda_{q} \lambda_{3} \lambda_{4}\right\}=\{L--\},\{L-+\},\{L+-\},\{L++\}$, which allows us to obtain the remaining ones for right-handed quarks by a simple charge-conjugation transformation, as we discuss below.

In order to compute the helicity amplitudes, we regulate the infrared and ultraviolet divergences using dimensional regularization, i.e., we work in $d=4-2 \epsilon$ dimensions. By choosing a gauge such that

$\epsilon_{i} \cdot p_{i}=0, \quad i=3,4, \quad$ and $\quad \epsilon_{3} \cdot p_{2}=\epsilon_{4} \cdot p_{1}=0$,

it is easy to see that, at any order in QCD perturbation theory, the Lorentz covariance dictates that the amplitude can be parameterized as

$$
\mathcal{A}(s, t)=\sum_{i=1}^{5} \mathcal{F}_{i}(s, t) \bar{u}\left(p_{2}\right) \Gamma_{i}^{\mu \nu} u\left(p_{1}\right) \epsilon_{3, \mu} \epsilon_{4, \nu},
$$

where $u$ and $\bar{u}$ are the spinors for the incoming quark and antiquark, respectively, and the five Lorentz tensors $\Gamma_{i}^{\mu \nu}$ are defined as

$$
\begin{gathered}
\Gamma_{1}^{\mu \nu}=\gamma^{\mu} p_{2}^{\nu}, \quad \Gamma_{2}^{\mu \nu}=\gamma^{\nu} p_{1}^{\mu}, \\
\Gamma_{3}^{\mu \nu}=\not{ }_{3} p_{1}^{\mu} p_{2}^{\nu}, \quad \Gamma_{4}^{\mu \nu}=\not p_{3} g^{\mu \nu}, \\
\text { and } \quad \Gamma_{5}^{\mu \nu}=\gamma^{\mu} \not p_{3} \gamma^{\nu} .
\end{gathered}
$$

The functions $\mathcal{F}_{i}(s, t)$ are scalar form factors that only depend on the Mandelstam invariants and on the number of dimensions $d$. Since the color structure is straightforward, we keep color indices implicit here. For ease of typing, we define the five independent structures as

$$
T_{i}=\bar{u}\left(p_{2}\right) \Gamma_{i}^{\mu \nu} u\left(p_{1}\right) \epsilon_{3, \mu} \epsilon_{4, \nu}
$$

and from now on, with a slight abuse of language, we refer to the $T_{i}$ as the independent "tensors" for the problem at hand.

At first sight, it may seem puzzling that we find five independent tensor structures when we have only four helicity amplitudes (not considering charge conjugation). This mismatch is easy to explain, however: the decomposition Eq. (5) is valid for arbitrary dimension $d$. For fourdimensional external states, the five tensor structures $T_{i}$ are no longer independent, and four of them are enough to span the whole space $[54,55]$. Since eventually we are only interested in the $d \rightarrow 4$ limit, it is convenient to reorganize the tensors $T_{i}$ and choose for $T_{5}$ a linear combination that is identically zero when four-dimensional external states are considered. This can be achieved by choosing

$$
\begin{aligned}
& \bar{T}_{i}=T_{i}, \quad i=1, \ldots, 4, \quad \text { and } \\
& \bar{T}_{5}=T_{5}-\frac{u}{s} T_{1}+\frac{u}{s} T_{2}-\frac{2}{s} T_{3}+T_{4} .
\end{aligned}
$$

We then write the scattering amplitude as

$$
\mathcal{A}(s, t)=\sum_{i=1}^{5} \overline{\mathcal{F}}_{i}(s, t) \bar{T}_{i},
$$

where the form factors $\overline{\mathcal{F}}_{i}$ are suitable linear combinations of the original $\mathcal{F}_{i}$, whose explicit form will be irrelevant in the following. All the nontrivial information for the process Eq. (1) is encoded in the form factors $\overline{\mathcal{F}}_{i}$. We stress once more that, while all five form factors are in general required for the result in conventional dimensional regularization, only the first four are enough to compute the helicity amplitudes in the 't Hooft-Veltman scheme (see the Supplemental Material [56] for more details).

Equation (9) can be inverted to select individual form factors. This is done by introducing suitable projector operators defined such that

$$
\sum_{\text {pol }} \mathcal{P}_{i} \mathcal{A}(s, t)=\overline{\mathcal{F}}_{i}(s, t),
$$

where we use $d$ dimensional polarization sums. \{Note that the individual form factors are not gauge invariant [see 
Eq. (4)], and the reference vector $q_{3(4)}=p_{2(1)}$ should be used in the polarization sums. $\}$ Since the five $\bar{T}_{i}$ form a basis for our space, we can write the projectors as

$$
\mathcal{P}_{i}=\sum_{k=1}^{5} c_{i k} \bar{T}_{k}^{\dagger}
$$

A straightforward calculation, reported in the Supplement Material [56], shows that the matrix $c_{\text {ik }}$ is block diagonal:

$$
c_{\mathrm{ik}}=\left[\begin{array}{cc}
C_{4 \times 4} & 0 \\
0 & c_{55}
\end{array}\right] \text {. }
$$

As a consequence, the fifth tensor $\bar{T}_{5}$ decouples from the other four. This, combined with the fact that the fifth tensor always evaluates to zero for four-dimensional external states, shows that the helicity amplitudes receive contributions only from the first four form factors in Eq. (9). In other words, there is a one-to-one correspondence between helicity amplitudes and form factors in the 't HooftVeltman scheme [57]. This statement is quite general, and it is always possible to construct a basis where the decoupling of tensors leading to vanishing results in $d=4$ is manifest. Details of how to achieve this in general will be presented elsewhere [55].

In order to compute the form factors, we generate the relevant tree-level and one-, two-, and three-loop Feynman diagrams with QGRAF [58] and apply to each of them the projectors defined in Eqs. (11) and (12). We perform all required color and Dirac algebra with FORM [59]. There are two diagrams at tree level, 10 at one loop, 143 at two loops, and 2922 at three loops. At a given number of loops, each Feynman diagram can be identified with a graph, which is specified by the permutation of the external legs and by the number and topology of the internal lines. In particular, at three loops, where the number of Feynman diagrams becomes rather large, it is convenient to sum together those diagrams that are associated with the same graph and manipulate them together to avoid as much as possible performing the same operation multiple times. Adopting these steps allows us to express the three-loop form factors in terms of a large number of scalar integrals of the form

$$
\mathcal{I}_{n_{1} \cdots n_{15}}^{\mathrm{fam}}=e^{3 \epsilon \gamma_{E}} \int \prod_{i=1}^{3} \frac{d^{d} k_{i}}{i \pi^{d / 2}} \frac{1}{D_{1}^{n_{1}} \cdots D_{15}^{n_{15}}}
$$

with $\gamma_{E} \approx 0.5772$ the Euler constant. [Note that $n_{i} \in \mathbb{Z}$, i.e., the integrals in Eq. (13) can have irreducible numerator structures.] The propagators $D_{j}$ can be drawn from three different families of integrals-fam $=\{$ PL, NPL1, NPL2 $\}-$ and their crossings. The definition of the three families is provided in the Supplemental Material [56]. A key feature of integrals of the form Eq. (13) is that they are not all independent. Instead, through a now-standard use of integration-by-parts identities, most of them can be expressed in terms of a much smaller set of so-called master integrals $[60,61]$. This can be done in principle algorithmically for any process [62] for integrals of the form of Eq. (13). Despite being well-understood in principle [62], this reduction step is computationally highly nontrivial for the problem at hand because it requires the symbolic solution of large systems of linear equations. We have achieved it through a combined use of the public code REDUZE2 $[63,64]$ and an in-house implementation, FINRED, that employs finite field sampling [65-68] and syzygy techniques [69-74]. In this way, the three-loop form factors $\overline{\mathcal{F}}_{j}^{(3)}(s, t)$ can be expressed as linear combinations of a total of 486 (132) independent master integrals, including (excluding) integrals related by crossings of the external legs.

A basis of master integrals, excluding integrals obtained from them by crossings of the external legs, has been computed for the first time in Ref. [50] using the method of differential equations [75-79] augmented by the choice of a canonical basis $[80,81]$. There it was shown that all these integrals can be expressed in terms of a particularly simple and well understood class of functions, harmonic polylogarithms [82-86]. The present calculation requires results through to transcendental weight six [87]. We have rederived the differential equations fulfilled by the master integrals, including all their nontrivial crossings required for the calculation of the actual scattering amplitudes. We have verified that the results of Ref. [50] fulfill the differential equations. As a check, we also have recomputed various boundary constants for the integrals of Ref. [50] independently. For manipulating the harmonic polylogarithms, we used in-house routines and PolyLogTools [88].

Extra care has to be taken in computing the boundary conditions and in analytically continuing the integrals to the physical scattering region in Eq. (3). Indeed, it is well known that scattering amplitudes are multivalued complex functions of the external kinematics and that, in order to obtain physical results, one must define the integrals on the correct Riemann sheet whenever one or more of the Mandelstam invariants are positive. The standard approach consists, whenever possible, of computing the relevant scattering amplitude for the unphysical values of the kinematics where all Mandelstam variables are negative and a real result is expected. If this is possible, it is then often simple to analytically continue the amplitudes to physical kinematics using the Feynman prescription $s_{i} \rightarrow s_{i}+i 0$ for each Mandelstam invariant $s_{i}$ that crosses a branch cut. Unfortunately, this approach fails for $2 \rightarrow 2$ massless scattering where the momentum conservation relation $s+t+u=0$ prevents us from finding such a unphysical region. As a consequence, the scattering amplitudes must be computed directly in a region where at least one Mandelstam invariant is positive. In the present case, extra care has to be taken since the boundary conditions provided in Ref. [50] are computed on the unphysical 
Riemann sheet reached by giving a small and negative imaginary part to the Mandelstam invariant $s \rightarrow s-i 0$. Of course, it is expected that these results should be related to the ones for $s \rightarrow s+i 0$ by complex conjugation. We have continued the results of Ref. [50] back to the physical Riemann sheet and checked that this holds. We then used them to obtain analytic expressions for all required crossings (see [89] for details). We used these integrals to compute the three-loop form factors $\overline{\mathcal{F}}_{j}^{(3)}(s, t)$.

The results computed with the procedure discussed so far contain ultraviolet and infrared singularities. Up to three loops, these can be written as follows:

$$
\overline{\mathcal{F}}_{i}=\delta_{\mathrm{kl}}(4 \pi \alpha) e_{q}^{2} \sum_{k=0}^{3}\left(\frac{\alpha_{s, b}}{2 \pi}\right)^{k} \overline{\mathcal{F}}_{i}^{(k, b)},
$$

where $e=\sqrt{4 \pi \alpha}$ is the electric charge, $e_{q}$ is the charge of the incoming quark in units of $e, \alpha_{s, b}$ is the bare strong coupling, and $\delta_{\mathrm{kl}}$ carries the color indices of the two incoming quarks. We remove ultraviolet singularities by expressing our result in terms of the $\overline{\mathrm{MS}}$ renormalized coupling $\alpha_{s}(\mu)$ :

$$
\overline{\mathcal{F}}_{i}=\delta_{\mathrm{kl}}(4 \pi \alpha) e_{q}^{2} \sum_{k=0}^{3}\left[\frac{\alpha_{s}(\mu)}{2 \pi}\right]^{k} \overline{\mathcal{F}}_{i}^{(k)} .
$$

The relation between renormalized and bare coupling is given by

$$
S_{\epsilon} \alpha_{s, b}=\mu^{2 \epsilon} \alpha_{s}(\mu) Z\left[\alpha_{s}(\mu)\right],
$$

with $S_{\epsilon}=(4 \pi)^{-\epsilon} e^{-\gamma_{E} \epsilon}$ and

$$
Z[\alpha]=1-\frac{\beta_{0}}{\epsilon}\left(\frac{\alpha_{s}}{2 \pi}\right)+\left(\frac{\beta_{0}^{2}}{\epsilon^{2}}-\frac{\beta_{1}}{2 \epsilon}\right)\left(\frac{\alpha_{s}}{2 \pi}\right)^{2}+\mathcal{O}\left(\alpha_{s}^{3}\right) .
$$

The explicit form of the $\beta$-function coefficient $\beta_{0,1}$ is reported in the Supplemental Material [56]. For definiteness, we will present our results for $\mu^{2}=s$. It is straightforward to obtain results at any other scale using renormalization-group arguments.

The renormalized form factors $\overline{\mathcal{F}}_{i}^{(k)}$ of Eq. (15) still contain infrared singularities. Their form is universal and can be expressed in terms of the lower-order scattering amplitudes as follows $[90,91]$ :

$$
\begin{aligned}
& \overline{\mathcal{F}}_{i}^{(1)}=\mathcal{I}_{1} \overline{\mathcal{F}}_{i}^{(0)}+\overline{\mathcal{F}}_{i}^{(1, \text { fin })}, \\
& \overline{\mathcal{F}}_{i}^{(2)}=\mathcal{I}_{2} \overline{\mathcal{F}}_{i}^{(0)}+\mathcal{I}_{1} \overline{\mathcal{F}}_{i}^{(1)}+\overline{\mathcal{F}}_{i}^{(2, \text { fin })}, \quad \text { and } \\
& \overline{\mathcal{F}}_{i}^{(3)}=\mathcal{I}_{3} \overline{\mathcal{F}}_{i}^{(0)}+\mathcal{I}_{2} \overline{\mathcal{F}}_{i}^{(1)}+\mathcal{I}_{1} \overline{\mathcal{F}}_{i}^{(2)}+\overline{\mathcal{F}}_{i}^{(3, \text { fin })} .
\end{aligned}
$$

In these equations, the $\mathcal{I}_{j}$ are universal factors that only depend on the center of mass energy of the colored partons $s$, on $\epsilon$ and on the QCD Casimirs $C_{F}=4 / 3, C_{A}=3$, as well as on the number of light fermions $n_{f}$. We spell them out explicitly in the Supplemental Material [56]. For our discussion, it is only important to note that the function $\mathcal{I}_{i}$ contains infrared poles up to order $2^{i}$, i.e., $\mathcal{I}_{1} \sim 1 / \epsilon^{2}$, $\mathcal{I}_{2} \sim 1 / \epsilon^{4}$, and $\mathcal{I}_{3} \sim 1 / \epsilon^{6}$. The finite remainders $\overline{\mathcal{F}}_{i}^{(k \text {,fin })}$ in Eq. (18) are finite in the $\epsilon \rightarrow 0$ limit and contain all the nontrivial physics information for the process Eq. (1).

As we have already mentioned, it is straightforward to obtain the helicity amplitudes from our form factors by evaluating the tensor structures $\bar{T}_{i}$ for well-defined helicity states [see Eq. (8)]. We stress once more that for any helicity configuration one has $\bar{T}_{5, \lambda_{q} \lambda_{3} \lambda_{4}}=0$. We write for left-handed spinors $\bar{u}_{L}\left(p_{2}\right)=\langle 2|$ and $\left.u_{L}\left(p_{1}\right)=\mid 1\right]$ and for the photon $j$ of momentum $p_{j}$

$$
\epsilon_{j,-}^{\mu}\left(q_{j}\right)=\frac{\left\langle q_{j}\left|\gamma^{\mu}\right| j\right]}{\sqrt{2}\left\langle q_{j} j\right\rangle} \quad \text { and } \quad \epsilon_{\mathrm{j},+}^{\mu}\left(\mathrm{q}_{\mathrm{j}}\right)=\frac{\left\langle\mathrm{j}\left|\gamma^{\mu}\right| \mathrm{q}_{\mathrm{j}}\right]}{\sqrt{2}\left[\mathrm{jq}_{\mathrm{j}}\right]} .
$$

With these definitions we find

$$
\begin{aligned}
& \mathcal{A}_{L--}=\frac{2[34]^{2}}{\langle 13\rangle[23]} \alpha(x), \quad \mathcal{A}_{L-+}=\frac{2\langle 24\rangle[13]}{\langle 23\rangle[24]} \beta(x), \\
& \mathcal{A}_{L+-}=\frac{2\langle 23\rangle[41]}{\langle 24\rangle[32]} \gamma(x), \quad \text { and } \quad \mathcal{A}_{\mathrm{L}++}=\frac{2\langle 34\rangle^{2}}{\langle 31\rangle[23]} \delta(\mathrm{x}),
\end{aligned}
$$

with

$$
\begin{aligned}
& \alpha(x)=\frac{t}{2}\left(\overline{\mathcal{F}}_{2}-\frac{t}{2} \overline{\mathcal{F}}_{3}+\overline{\mathcal{F}}_{4}\right), \\
& \beta(x)=\frac{t}{2}\left(\frac{s}{2} \overline{\mathcal{F}}_{3}+\overline{\mathcal{F}}_{4}\right), \\
& \gamma(x)=\frac{s t}{2 u}\left(\overline{\mathcal{F}}_{2}-\overline{\mathcal{F}}_{1}-\frac{t}{2} \overline{\mathcal{F}}_{3}-\frac{t}{s} \overline{\mathcal{F}}_{4}\right), \quad \text { and } \\
& \delta(x)=\frac{t}{2}\left(\overline{\mathcal{F}}_{1}+\frac{t}{2} \overline{\mathcal{F}}_{3}-\overline{\mathcal{F}}_{4}\right) .
\end{aligned}
$$

Note that the remaining amplitudes for right-handed quarks can be obtained by a charge-conjugation transformation as follows:

$$
\mathcal{A}_{R \lambda_{3} \lambda_{4}}=\mathcal{A}_{L \lambda_{3}^{*} \lambda_{4}^{*}}(\langle i j\rangle \leftrightarrow[j i]),
$$

where $\lambda_{i}^{*}$ indicates the opposite helicity of $\lambda_{i}$. Symmetry under the exchange of the two photons requires

$\gamma(x)=\beta(1-x), \quad \delta(x)=-\alpha(x), \quad$ and $\quad \alpha(1-\mathrm{x})=-\alpha(\mathrm{x})$,

and at tree level we find $\alpha=\delta=0$ and $\beta=\gamma=1$. 
Using the relations Eqs. (19) and (20), we obtain the three-loop renormalized finite remainders $\alpha^{(3, \text { fin })}, \beta^{(3, \text { fin })}$, $\gamma^{(3, \text { fin })}$, and $\delta^{(3, \text { fin) }}$ defined in analogy to Eqs. (15) and (18). This represents the first three-loop calculation of a full fourpoint QCD amplitude. Our result can be expressed in terms of harmonic polylogarithms of weights 0 and 1, or, alternatively, in terms of a more compact functional basis consisting of 14 classical polylogarithms and the nine functions

$$
\begin{aligned}
& \operatorname{Li}_{3,2}(x, 1), \quad \operatorname{Li}_{3,2}(1-x, 1), \quad \operatorname{Li}_{3,2}(1, x), \\
& \mathrm{Li}_{3,3}(x, 1), \quad \operatorname{Li}_{3,3}(1-x, 1), \quad \operatorname{Li}_{3,3}(x /(x-1), 1) \text {, } \\
& \mathrm{Li}_{4,2}(x, 1), \quad \mathrm{Li}_{4,2}(1-x, 1), \quad \text { and } \quad \operatorname{Li}_{2,2,2}(\mathrm{x}, 1,1) \text {, }
\end{aligned}
$$

which are two and threefold nested sums in the conventions of [84]. (Note that PolyLogTools [88] uses a convention for indices and arguments different from the ones we adopt here.) In the latter representation, our three-loop amplitudes can be evaluated in fractions of a second in one phase-space point. Although the result is relatively compact, it is too long to be presented here. We have included it in electronic format in the ancillary files of this Letter. In Fig. 1, we show a numerical evaluation of our analytic result for all independent finite remainder functions through to three loops. While only the real parts are shown, we note that the imaginary parts of the loop corrections can actually exceed their real parts substantially in the case of $\beta$.

Before concluding, we list some of the checks that we have performed to verify the correctness of our calculation. We have performed the tree-level and one- and two-loop computations from scratch using our setup and obtained agreement for the finite reminders at $\mathcal{O}\left(\epsilon^{0}\right)$ with results in the literature [10]. From Eq. (18) it follows that, to extract $\overline{\mathcal{F}}_{i}^{(3, \text { fin })}$, one must expand the one- and two-loop results up to orders $\epsilon^{4}$ and $\epsilon^{2}$, respectively. To check the correctness of our one- and two-loop results, we have abelianized the result of [49] and checked it against the abelian part of our results up to weight six. As we have mentioned, Bose symmetry relates amplitudes of different helicities. We have verified that our helicity amplitude coefficients up to three loops display the Bose symmetry properties in Eq. (22).

A nontrivial aspect of our calculation is the analytic continuation of all the required integrals to the physical region. To check our procedure, we verified that our solutions are consistent with the boundary values for the master integrals that can be inferred by imposing regularity conditions on their differential equations. This allowed us to relate complicated four-point integrals to simpler two- and three-point ones (see [92] for a compilation), whose analytic continuation is straightforward. Moreover, we used REDUZE2 to find nontrivial symmetry relations among the master integrals and their crossings and verified that they are all were satisfied by our analytic results. In addition, we checked the finite part of the twoloop integrals against the literature and some of the simple three-loop integrals against SECDEC [93]. Finally, the most powerful check of the correctness of our result is that the remainders $\overline{\mathcal{F}}_{i}^{(3, \text { fin })}$ are in fact finite in the $\epsilon \rightarrow 0$ limit. This also tests in a nontrivial way our analytic continuation procedure, as it links three-loop integrals with lower loop ones.

In conclusion, in this Letter we have reported the first calculation of a three-loop four-point scattering amplitude in full QCD. We have obtained our results by defining a minimal set of projectors that allowed us to extract all the information required to reconstruct the helicity amplitudes. Our result can be expressed in terms of classical polylogarithms plus a handful of multiple polylogarithms, and it is very compact for a QCD amplitude of this type. The methods we employed for this calculation are generic and can be used to compute the three-loop helicity amplitudes for $2 \rightarrow 2$ scattering of massless partons in QCD. We leave this for future investigations.
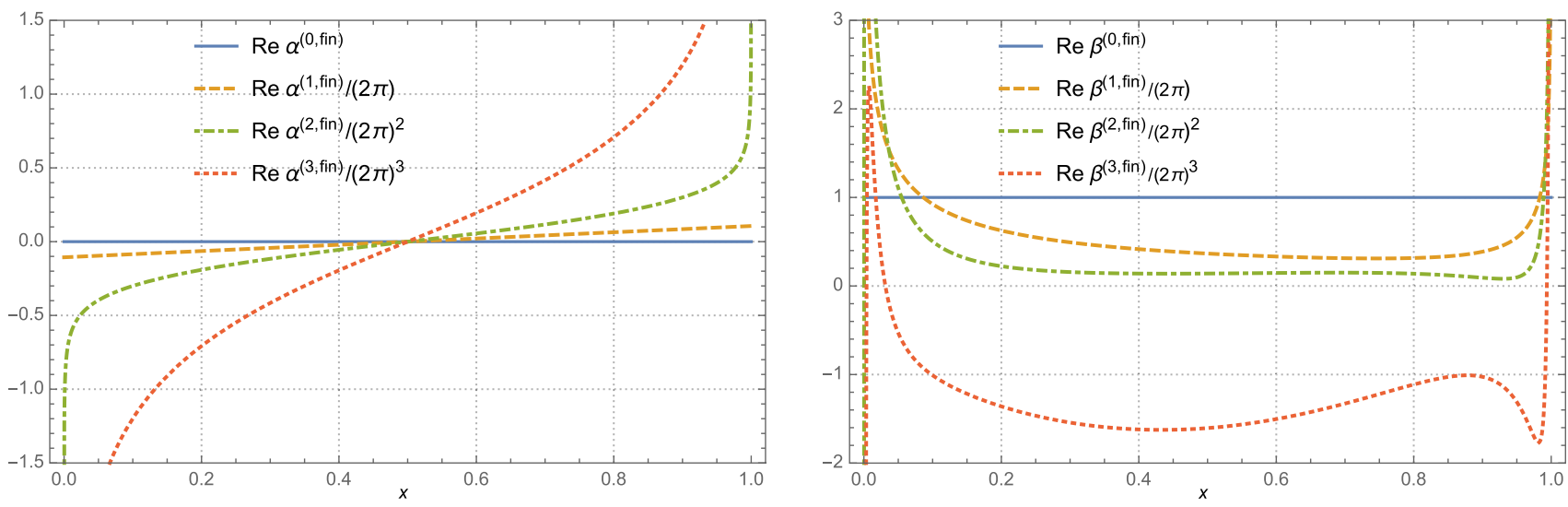

FIG. 1. The real part of the three-loop finite remainder functions $\alpha^{(3) \text {,fin }}(x)$ and $\beta^{(3) \text {,fin }}(x)$ that determine the helicity amplitudes $\mathcal{A}_{L--}$ and $\mathcal{A}_{L-+}$, respectively, for $u \bar{u} \rightarrow \gamma \gamma$ and $\mu^{2}=s$. 
We are grateful to $\mathrm{P}$. Bargiela for providing several nontrivial checks of the one- and two-loop helicity amplitudes at higher orders in the dimensional regulator $\epsilon$. L. T. is grateful to T. Peraro for many clarifying discussions on the general idea behind the tensor decomposition for scattering amplitudes used in this Letter. We also thank T. Peraro for various comments on the manuscript and $\mathrm{B}$. Mistlberger for clarifications on the results of $[49,50]$. The research of F. C. is partially supported by the ERC Starting Grant No. 804394 HipQCD. A. v. M. is supported in part by the National Science Foundation through Grants No. 1719863 and No. 2013859. L. T. is supported by the Royal Society through Grant No. URF/R1/191125.

*Corresponding author.

fabrizio.caola@physics.ox.ac.uk

Corresponding author.

vmante@msu.edu

"Corresponding author.

lorenzo.tancredi@physics.ox.ac.uk

[1] D. de Florian et al. (LHC Higgs Cross Section Working Group Collaboration), Handbook of LHC Higgs cross sections: 4. Deciphering the nature of the Higgs sector, arXiv: 1610.07922.

[2] M. Aaboud et al. (ATLAS Collaboration), Measurement of the $W$-boson mass in pp collisions at $\sqrt{s}=7 \mathrm{TeV}$ with the ATLAS detector, Eur. Phys. J. C 78, 110 (2018); Erratum, Eur. Phys. J. C 78, 898 (2018).

[3] R. D. Ball et al. (NNPDF Collaboration), Parton distributions from high-precision collider data, Eur. Phys. J. C 77, 663 (2017).

[4] A. M. Sirunyan et al. (CMS Collaboration), Measurements of $\mathrm{pp} \rightarrow \mathrm{ZZ}$ production cross sections and constraints on anomalous triple gauge couplings at $\sqrt{s}=13 \mathrm{TeV}$, arXiv:2009.01186.

[5] A. M. Sirunyan et al. (CMS Collaboration), $\mathrm{W}^{+} \mathrm{W}^{-}$boson pair production in proton-proton collisions at $\sqrt{s}=13 \mathrm{TeV}$, Phys. Rev. D 102, 092001 (2020).

[6] ATLAS Collaboration, Standard model summary plots spring, Report No. ATL-PHYS-PUB-2020-010, 2020.

[7] ATLAS Collaboration, Combination of ATLAS and CMS top-quark pair cross-section measurements using protonproton collisions at $\sqrt{s}=8 \mathrm{TeV}$, Report No. ATLASCONF-2014-054, 2014.

[8] L. Garland, T. Gehrmann, E. Glover, A. Koukoutsakis, and E. Remiddi, Two loop QCD helicity amplitudes for $e^{+} e^{-} \rightarrow 3 \sim$ jets, Nucl. Phys. B642, 227 (2002).

[9] C. Anastasiou, E. W. N. Glover, and M. Tejeda-Yeomans, Two loop QED and QCD corrections to massless fermion boson scattering, Nucl. Phys. B629, 255 (2002).

[10] E. Glover and M. Tejeda-Yeomans, Two loop QCD helicity amplitudes for massless quark massless gauge boson scattering, J. High Energy Phys. 06 (2003) 033.

[11] E. Glover, Two loop QCD helicity amplitudes for massless quark quark scattering, J. High Energy Phys. 04 (2004) 021.

[12] Z. Bern, A. De Freitas, and L. J. Dixon, Two loop amplitudes for gluon fusion into two photons, J. High Energy Phys. 09 (2001) 037.
[13] Z. Bern, A. De Freitas, and L. J. Dixon, Two loop helicity amplitudes for gluon-gluon scattering in QCD and supersymmetric Yang-Mills theory, J. High Energy Phys. 03 (2002) 018.

[14] Z. Bern, A. De Freitas, and L. J. Dixon, Two loop helicity amplitudes for quark gluon scattering in QCD and gluino gluon scattering in supersymmetric Yang-Mills theory, J. High Energy Phys. 06 (2003) 028; Erratum, J. High Energy Phys. 04 (2014) 112.

[15] T. Gehrmann and L. Tancredi, Two-loop QCD helicity amplitudes for $q \bar{q} \rightarrow W^{ \pm} \gamma$ and $q \bar{q} \rightarrow Z^{0} \gamma$, J. High Energy Phys. 02 (2012) 004.

[16] T. Gehrmann, M. Jaquier, E. Glover, and A. Koukoutsakis, Two-loop QCD corrections to the helicity amplitudes for $H \rightarrow 3$ partons, J. High Energy Phys. 02 (2012) 056.

[17] T. Gehrmann, L. Tancredi, and E. Weihs, Two-loop QCD helicity amplitudes for $g g \rightarrow Z g$ and $g g \rightarrow Z \gamma$, J. High Energy Phys. 04 (2013) 101.

[18] F. Caola, J. M. Henn, K. Melnikov, A. V. Smirnov, and V. A. Smirnov, Two-loop helicity amplitudes for the production of two off-shell electroweak bosons in quark-antiquark collisions, J. High Energy Phys. 11 (2014) 041.

[19] F. Caola, J. M. Henn, K. Melnikov, A. V. Smirnov, and V. A. Smirnov, Two-loop helicity amplitudes for the production of two off-shell electroweak bosons in gluon fusion, J. High Energy Phys. 06 (2015) 129.

[20] T. Gehrmann, A. von Manteuffel, and L. Tancredi, The twoloop helicity amplitudes for $q \bar{q}^{\prime} \rightarrow V_{1} V_{2} \rightarrow 4$ leptons, J. High Energy Phys. 09 (2015) 128.

[21] A. von Manteuffel and L. Tancredi, The two-loop helicity amplitudes for $g g \rightarrow V_{1} V_{2} \rightarrow 4$ leptons, J. High Energy Phys. 06 (2015) 197.

[22] R. Bonciani, A. Ferroglia, T. Gehrmann, A. von Manteuffel, and C. Studerus, Two-loop leading color corrections to heavy-quark pair production in the gluon fusion channel, J. High Energy Phys. 01 (2011) 102.

[23] P. Bärnreuther, M. Czakon, and P. Fiedler, Virtual amplitudes and threshold behaviour of hadronic top-quark pairproduction cross sections, J. High Energy Phys. 02 (2014) 078.

[24] S. Borowka, N. Greiner, G. Heinrich, S. Jones, M. Kerner, J. Schlenk, U. Schubert, and T. Zirke, Higgs Boson Pair Production in Gluon Fusion at Next-to-Leading Order with Full Top-Quark Mass Dependence, Phys. Rev. Lett. 117, 012001 (2016); Erratum, Phys. Rev. Lett. 117, 079901 (2016).

[25] K. Melnikov, L. Tancredi, and C. Wever, Two-loop amplitudes for $q g \rightarrow H q$ and $q \bar{q} \rightarrow H g$ mediated by a nearly massless quark, Phys. Rev. D 95, 054012 (2017).

[26] K. Kudashkin, K. Melnikov, and C. Wever, Two-loop amplitudes for processes $g g \rightarrow H g, \quad q g \rightarrow H q$ and $q \bar{q} \rightarrow \mathrm{Hg}$ at large Higgs transverse momentum, J. High Energy Phys. 02 (2018) 135.

[27] J. Baglio, F. Campanario, S. Glaus, M. Mühlleitner, M. Spira, and J. Streicher, Gluon fusion into Higgs pairs at NLO QCD and the top mass scheme, Eur. Phys. J. C 79, 459 (2019).

[28] M. Heller, A. von Manteuffel, and R. M. Schabinger, Multiple polylogarithms with algebraic arguments and the two-loop EW-QCD Drell-Yan master integrals, Phys. Rev. D 102, 016025 (2020). 
[29] H. Frellesvig, M. Hidding, L. Maestri, F. Moriello, and G. Salvatori, The complete set of two-loop master integrals for Higgs + jet production in QCD, J. High Energy Phys. 06 (2020) 093.

[30] C. Brønnum-Hansen and C.-Y. Wang, Contribution of third generation quarks to two-loop helicity amplitudes for W boson pair production in gluon fusion, J. High Energy Phys. 01 (2021) 170.

[31] S. Badger, C. Broennum-Hansen, H. B. Hartanto, and T. Peraro, First Look at Two-Loop Five-Gluon Scattering in QCD, Phys. Rev. Lett. 120, 092001 (2018).

[32] S. Abreu, F. F. Cordero, H. Ita, B. Page, and M. Zeng, Planar Two-loop five-gluon amplitudes from numerical unitarity, Phys. Rev. D 97, 116014 (2018).

[33] S. Abreu, J. Dormans, F. F. Cordero, H. Ita, and B. Page, Analytic Form of Planar Two-Loop Five-Gluon Scattering Amplitudes in QCD, Phys. Rev. Lett. 122, 082002 (2019).

[34] S. Abreu, F. F. Cordero, H. Ita, B. Page, and V. Sotnikov, Planar two-loop five-parton amplitudes from numerical unitarity, J. High Energy Phys. 11 (2018) 116.

[35] D. Chicherin, T. Gehrmann, J. M. Henn, P. Wasser, Y. Zhang, and S. Zoia, Analytic Result for a Two-Loop FiveParticle Amplitude, Phys. Rev. Lett. 122, 121602 (2019).

[36] S. Badger, C. Brønnum-Hansen, H. B. Hartanto, and T. Peraro, Analytic helicity amplitudes for two-loop five-gluon scattering: The single-minus case, J. High Energy Phys. 01 (2019) 186.

[37] S. Badger, D. Chicherin, T. Gehrmann, G. Heinrich, J. Henn, T. Peraro, P. Wasser, Y. Zhang, and S. Zoia, Analytic form of the Full Two-Loop Five-Gluon All-Plus Helicity Amplitude, Phys. Rev. Lett. 123, 071601 (2019).

[38] D. Chicherin, T. Gehrmann, J. M. Henn, P. Wasser, Y. Zhang, and S. Zoia, The two-loop five-particle amplitude in $\mathcal{N}=8$ supergravity, J. High Energy Phys. 03 (2019) 115.

[39] H. B. Hartanto, S. Badger, C. Brønnum-Hansen, and T. Peraro, A numerical evaluation of planar two-loop helicity amplitudes for a W-boson plus four partons, J. High Energy Phys. 09 (2019) 119.

[40] S. Abreu, B. Page, E. Pascual, and V. Sotnikov, Leadingcolor two-loop QCD corrections for three-photon production at hadron colliders, J. High Energy Phys. 01 (2021) 78.

[41] G. De Laurentis and D. Maître, Two-loop five-parton leading-colour finite remainders in the spinor-helicity formalism, J. High Energy Phys. 02 (2021) 016.

[42] H. A. Chawdhry, M. L. Czakon, A. Mitov, and R. Poncelet, NNLO QCD corrections to three-photon production at the LHC, J. High Energy Phys. 02 (2020) 057.

[43] S. Kallweit, V. Sotnikov, and M. Wiesemann, Triphoton production at hadron colliders in NNLO QCD, Phys. Lett. B 812, 136013 (2021).

[44] J. M. Henn and B. Mistlberger, Four-Gluon Scattering at Three Loops, Infrared Structure, and the Regge Limit, Phys. Rev. Lett. 117, 171601 (2016).

[45] J. M. Henn and B. Mistlberger, Four-graviton scattering to three loops in $\mathcal{N}=8$ supergravity, J. High Energy Phys. 05 (2019) 023.

[46] S. Moch, J. Vermaseren, and A. Vogt, Three-loop results for quark and gluon form-factors, Phys. Lett. B 625, 245 (2005).
[47] P. Baikov, K. Chetyrkin, A. Smirnov, V. Smirnov, and M. Steinhauser, Quark and Gluon Form Factors to Three Loops, Phys. Rev. Lett. 102, 212002 (2009).

[48] T. Gehrmann, E. Glover, T. Huber, N. Ikizlerli, and C. Studerus, Calculation of the quark and gluon form factors to three loops in QCD, J. High Energy Phys. 06 (2010) 094.

[49] T. Ahmed, J. Henn, and B. Mistlberger, Four-particle scattering amplitudes in QCD at NNLO to higher orders in the dimensional regulator, J. High Energy Phys. 12 (2019) 177.

[50] J. Henn, B. Mistlberger, V. A. Smirnov, and P. Wasser, Constructing d-log integrands and computing master integrals for three-loop four-particle scattering, J. High Energy Phys. 04 (2020) 167.

[51] Z. Bern, L.J. Dixon, and C. Schmidt, The Diphoton background to a light Higgs boson at the LHC, Nucl. Phys. B, Proc. Suppl. 116, 178 (2003).

[52] S. Catani, L. Cieri, D. de Florian, G. Ferrera, and M. Grazzini, Diphoton Production at Hadron Colliders: A Fully-Differential QCD Calculation at NNLO, Phys. Rev. Lett. 108, 072001 (2012).

[53] T. Gehrmann, N. Glover, A. Huss, and J. Whitehead, Scale and isolation sensitivity of diphoton distributions at the LHC, J. High Energy Phys. 01 (2021) 108.

[54] T. Peraro and L. Tancredi, Physical projectors for multi-leg helicity amplitudes, J. High Energy Phys. 07 (2019) 114.

[55] T. Peraro and L. Tancredi, Tensor decomposition for bosonic and fermionic scattering amplitudes, arXiv: 2012.00820.

[56] See Supplemental Material at http://link.aps.org/ supplemental/10.1103/PhysRevLett.126.112004 for the analytic results of the calculation presented in this Letter in electronic format.

[57] G. 't Hooft and M.J.G. Veltman, Regularization and renormalization of gauge fields, Nucl. Phys. B44, 189 (1972).

[58] P. Nogueira, Automatic Feynman graph generation, J. Comput. Phys. 105, 279 (1993).

[59] J. Vermaseren, New features of FORM, arXiv:math-ph/ 0010025.

[60] F. Tkachov, A theorem on analytical calculability of four loop renormalization group functions, Phys. Lett. 100B, 65 (1981).

[61] K. Chetyrkin and F. Tkachov, Integration by Parts: The algorithm to calculate beta functions in 4 loops, Nucl. Phys. B192, 159 (1981).

[62] S. Laporta, High precision calculation of multiloop Feynman integrals by difference equations, Int. J. Mod. Phys. A 15, 5087 (2000).

[63] A. von Manteuffel and C. Studerus, Reduze 2-Distributed Feynman integral reduction, arXiv:1201.4330.

[64] C. Studerus, Reduze-Feynman integral reduction in $\mathrm{C}++$, Comput. Phys. Commun. 181, 1293 (2010).

[65] A. von Manteuffel and R. M. Schabinger, A novel approach to integration by parts reduction, Phys. Lett. B 744, 101 (2015).

[66] A. von Manteuffel and R. M. Schabinger, Quark and gluon form factors to four-loop order in QCD: The $N_{f}^{3}$ contributions, Phys. Rev. D 95, 034030 (2017). 
[67] T. Peraro, Scattering amplitudes over finite fields and multivariate functional reconstruction, J. High Energy Phys. 12 (2016) 030.

[68] T. Peraro, FiniteFlow: Multivariate functional reconstruction using finite fields and dataflow graphs, J. High Energy Phys. 07 (2019) 031.

[69] J. Gluza, K. Kajda, and D. A. Kosower, Towards a basis for planar two-loop integrals, Phys. Rev. D 83, 045012 (2011).

[70] R. M. Schabinger, A new algorithm for the generation of unitarity-compatible integration by parts relations, J. High Energy Phys. 01 (2012) 077.

[71] H. Ita, Two-loop integrand decomposition into master integrals and surface terms, Phys. Rev. D 94, 116015 (2016).

[72] K. J. Larsen and Y. Zhang, Integration-by-parts reductions from unitarity cuts and algebraic geometry, Phys. Rev. D 93, 041701(R) (2016).

[73] J. Böhm, A. Georgoudis, K. J. Larsen, M. Schulze, and Y. Zhang, Complete sets of logarithmic vector fields for integration-by-parts identities of Feynman integrals, Phys. Rev. D 98, 025023 (2018).

[74] B. Agarwal and A. Von Manteuffel, On the two-loop amplitude for $g g \rightarrow Z Z$ production with full top-mass dependence, Proc. Sci., RADCOR2019 (2019) 008 [arXiv:1912.08794].

[75] Z. Bern, L. J. Dixon, and D. A. Kosower, Dimensionally regulated pentagon integrals, Nucl. Phys. B412, 751 (1994).

[76] A. Kotikov, Differential equations method: New technique for massive Feynman diagrams calculation, Phys. Lett. B 254, 158 (1991).

[77] E. Remiddi, Differential equations for Feynman graph amplitudes, Nuovo Cimento A 110, 1435 (1997).

[78] T. Gehrmann and E. Remiddi, Differential equations for two loop four point functions, Nucl. Phys. B580, 485 (2000).

[79] A. Primo and L. Tancredi, On the maximal cut of Feynman integrals and the solution of their differential equations, Nucl. Phys. B916, 94 (2017).

[80] A. Kotikov, The property of maximal transcendentality in the $N=4$ supersymmetric Yang-Mills, arXiv:1005.5029.
[81] J. M. Henn, Multiloop Integrals in Dimensional Regularization Made Simple, Phys. Rev. Lett. 110, 251601 (2013).

[82] A. B. Goncharov, Multiple Polylogarithms, Cyclotomy and Modular Complexes, Math. Res. Lett. 5, 497 (1998).

[83] E. Remiddi and J. Vermaseren, Harmonic polylogarithms, Int. J. Mod. Phys. A 15, 725 (2000).

[84] J. Vollinga and S. Weinzierl, Numerical evaluation of multiple polylogarithms, Comput. Phys. Commun. 167, 177 (2005).

[85] C. Duhr, H. Gangl, and J. R. Rhodes, From polygons and symbols to polylogarithmic functions, J. High Energy Phys. 10 (2012) 075.

[86] C. Duhr, Hopf algebras, coproducts and symbols: An application to Higgs boson amplitudes, J. High Energy Phys. 08 (2012) 043.

[87] Note that a simple counting argument shows that there can be at most 146 functions that can contribute at weight six. This puts an upper bound on the number of independent master integrals that can contribute to the finite part of the three-loop amplitude.

[88] C. Duhr and F. Dulat, PolyLogTools-Polylogs for the masses, J. High Energy Phys. 08 (2019) 135.

[89] C. Anastasiou, T. Gehrmann, C. Oleari, E. Remiddi, and J. Tausk, The tensor reduction and master integrals of the two loop massless crossed box with lightlike legs, Nucl. Phys. B580, 577 (2000).

[90] S. Catani, The singular behavior of QCD amplitudes at two loop order, Phys. Lett. B 427, 161 (1998).

[91] T. Becher and M. Neubert, On the structure of infrared singularities of gauge-theory amplitudes, J. High Energy Phys. 06 (2009) 081.

[92] A. von Manteuffel, E. Panzer, and R. M. Schabinger, On the computation of form factors in massless QCD with finite master integrals, Phys. Rev. D 93, 125014 (2016).

[93] S. Borowka, G. Heinrich, S. P. Jones, M. Kerner, J. Schlenk, and T. Zirke, SecDec-3.0: Numerical evaluation of multiscale integrals beyond one loop, Comput. Phys. Commun. 196, 470 (2015). 